\title{
QoE in 5G Cloud Networks using Multimedia Services
}

\author{
Muhammad Sajid Mushtaq, Scott Fowler, Brice Augustin and Abdelhamid Mellouk
}

Conference Publication

\section{Tweet}

N.B.: When citing this work, cite the original article.

Original Publication:

Muhammad Sajid Mushtaq, Scott Fowler, Brice Augustin and Abdelhamid Mellouk, QoE in 5G Cloud Networks using Multimedia Services, 2016, IEEE Wireless Communication and Networking Conference (WCNC'16).

Copyright: www.iee.org

Postprint available at: Linköping University Electronic Press

http://urn.kb.se/resolve?urn=urn:nbn:se:liu:diva-128164

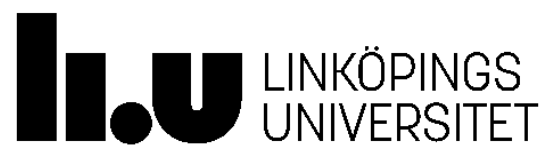




\title{
QoE in 5G Cloud Networks using Multimedia Services
}

\author{
M. Sajid Mushtaq*, Scott Fowler ${ }^{\dagger}$, Brice Augustin*, Abdelhamid Mellouk* \\ ${ }^{*}$ LiSSi/TINC Laboratory, Dept. of Networks and Telecoms, University of Paris-Est Créteil VdM (UPEC), France \\ ${ }^{\dagger}$ Mobile Telecoms., Department of Science \& Technology, Linköping University, Norrköping, Sweden
}

\begin{abstract}
The 4G standard Long Term Evolution-Advanced (LTE-A) has been deployed in many countries. Now, technology is evolving towards the $5 \mathrm{G}$ standard since it is expecting to start its service in $\mathbf{2 0 2 0}$. The $\mathbf{5 G}$ cellular networks will mainly contain in cloud computing and primarily Quality of Service (QoS) parameters (e.g. delay, loss rate, etc.) influence the cloud network performance. The impact of user perceived Quality of Experience (QoE) using multimedia services, and application significantly relies on the QoS parameters. The key challenge of $5 \mathrm{G}$ technology is to reduce the delay less than one millisecond. In this paper, we have described a method that minimizes the overall network delay for multimedia services; which are constant bit rate (VoIP) and variable bit rate (video) traffic model. We also proposed a method that measures the user's QoE for video streaming traffic using the network QoS parameters, i.e. delay and packet loss rate. The performance of proposed QoE method is compared with QoV method, and our proposed QoE method performs best by carefully handle the impact of QoS parameters. The results show that our described method successfully reduces the overall network delays, which result to maximize the user's QoE.
\end{abstract}

Keywords: QoE; QoS; 5G; Cloud; VoIP; Video; Multimedia Services.

\section{INTRODUCTION}

In the last two decades, we have witness the tremendous growth in wireless technology. According to Cisco forecast report, the global mobile data traffic will increase nearly by 11-fold in 2018 [1]. The multimedia traffic will be the main contributor over wireless communication system. It becomes important for the next-generation wireless networks $(5 \mathrm{G})$, to provide these multimedia services in an efficient way in order to cater the end users' quality expectation. Cloud computing will be fundamental part of $5 \mathrm{G}$ architecture that provides powerful computing platform to support ultra high-definition video services (e.g. Live IPTV, 2D/3D video, Video on Demand "VoD", Interactive gaming, etc.) to fulfil the demand of end users.

Cloud computing is a computing paradigm in which a configurable shared resources are available to fulfil the required demand (e.g. applications, services, storage, etc.). The main features of cloud computing consists of on-demand selfservice, resource pooling, broadband network access, rapid elasticity, and measured service [2]. It contains three main service models, Infrastructure-as-a-Service (IaaS), Platformas-a-Service(PaaS), and Software-as-a-Service (SaaS). With the enormous advance in wireless communication, smart devices (e.g. Tablet, iPhone, Android, Window, etc.) provide more flexibility to users via cloud computing system. This new paradigm of cloud computing in mobile environment introduces the Mobile Cloud Computing (MCC).

The mobile devices are still not sufficient powerful, because they have limited computing, storage, and energy resources. The MCC can effectively fill the gap between demanding computing power and mobile device limitations. MCC facilitates the mobile devices to offload the computing power and data storage demand into the powerful computing infrastructure of the cloud [3]. The future wireless $5 \mathrm{G}$ standards are still not completely finalized, but there some key visionary elements that will evolve the technologies. Cloud-based Radio Access Network (C-RAN) infrastructure is a novel cellular network architecture for $5 \mathrm{G}$ mobile networks, that will provide the shared on-demand computation, storage and network capacity wherever needed. In this paper, mobile device, user equipment and user are interchanged refer to same context's meaning.

Conventionally, the radio equipments such as Remote Radio-Unit (RRU) and Base-Band processing Unit (BBU), are located within the cellular Base Station (BS). To improve on the conventional method, C-RAN has been introduced. While C-RAN manages hundreds to thousands of remotely distributed RRU that connected to the centralized pool of BBUs. There are some significant advantages from distributed to centralized base-band processing. The consolidation, pooling, and virtualization of BBUs resources leads to lower deployment cost by avoiding the redundant capabilities and over-commissioning. It also enables the better load sharing, improved network performance via advanced processing techniques, saving equipment cost, and last but not the least reduce power consumption.

Generally, the performance of network is mainly depend on the network Quality of Service (QoS) parameters, that play important role in selecting the suitable server to improve the user satisfaction. In this paper, we describe the C-RAN that provides the multimedia video streaming and VoIP service to the end users. The impact of network QoS parameters, such as delay and packet loss rate are considered as the contributing factors that influence the user perceived Quality of Experience (QoE). We described an analytical method that decreases the overall network delay. The proposed QoE method measures the user's QoE using the delay and packet loss rate for video streaming service that consider the future needs of $5 \mathrm{G}$. Among the different factors, the network QoS parameters directly influence the end user perceived QoE. The main objective is 
to satisfy the user's QoE by reducing the traffic delay that can cause dropping of video packet in cloud network.

\section{RELATED WORK}

There are key influence factors that directly or indirectly affect the user's QoE. In wireless communication systems, the network QoS parameters (bandwidth, delay, packet loss rate, etc.) are very important that regulate the user perceived quality experience.

Generally, the server selection approaches are based on QoS measurement that considered the performance of network and server parameters. In [4], authors designed and evaluated the different server selection methods for VoD services using the video replication approach on different server. These methods investigated on a basic policy to request the service to the geographically closer server for mitigating the delay impact. In a similar manner, the proposed method in [5] always selects the closer server, which is based on the number of hop-count on the network path between the client and the server. In [6] authors proposed the server selection method that take into account the load information of each server, while in [7] authors proposed the delay based method that used the Round Trip Time (RTT) between the client and the servers to choose the suitable server.

As seen that the server selection methods considered different QoS parameters in order to select the appropriate server in the cloud networks such as delay, packet loss rate, Round Trip Time (RTT), hop-count between the client and the server, etc. However, these proposed approaches did not consider the important concept that find out the essential factors in the success of future services that are based on user's QoE. In [8], authors proposed the server selection method that considered the user perception to select the suitable server. The authors used the network QoS parameters to compute the user's QoE in terms of User Perception Score (UPS) using the PseudoSubjective Quality Assessment (PSQA) method [9].

In this paper, our proposed QoE method uses the QoS parameters (delay and packet loss rate) to calculate the user's QoE for video streaming traffic. The objective is to reduce the delay between the User Equipment (UE) and the cloud server by minimizing the network delay and maximize the user perception. The server selection is based on the QoE

\section{Cloud based Future Cellular Network}

The detail technical approaches in future 5G network are still not clear, because 5G standards have not been fix, but many things are cleared, e.g. support a 1000-fold gain in capacity, at least 100 billion devices are connected, and provide $10 \mathrm{Gbps}$ connection to individual user [10]. The core network will more base on cloud for both control purpose and data. Figure 1, illustrates the envisioned architecture of future cellular network (5G) along with cloud computing infrastructure. Generally, the traditional Base Station (BS) contains in two fundamental radio equipment i.e. Remote Radio Unit (RRU) and Base-Band process unit (BBU) located at the tower. The RRU handles the air interface, and BBU deals the data modulation and coding processing by controlling the connection between UE and cell. The future $5 \mathrm{G}$ cellular network, introduces the Cloud-Radio Access Network (C-RAN) infrastructure that shift the distributed processing units(i.e. $\mathrm{BBU}$ ) into the centralized. The BSs in C-RAN simplifies the network architecture, because all the signal processing and decision making are made by the wirelss cloud network. The IP traffic splitter proxy likes a System Architecture EvolutionGateway (SAE-GW) in Long Term Evolution (LTE) architecture where users data packets are passed to SAE-GW before sending to the Internet [11].

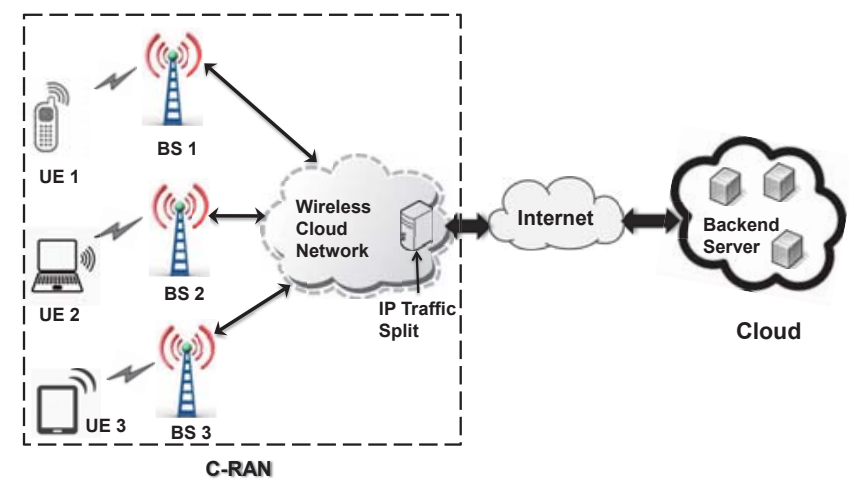

Fig. 1: Envisioned Future Cellular Network Architecture

\section{TRAFFIC MODEL}

The network can be modelled as a graph $G(V, A)$, where $V$ is the set of all vertices (e.g. in our case UEs and eNodeBs), while $\mathrm{A}$ is the set of arcs (represents the possible wireless transmission between the vertices) in the network. Each vertex $v \in V$ and each $\operatorname{arc} \in A$. An arc in $A$ can be characterized as arc $(i, j)$ with two parameters $i$ ) link service capacity, and $i i)$ user's QoE, which can be modelled as a general queueing system. The model considers the link as an average data rate $\left(\lambda_{i j}\right)$ and services capacity $\left(\mu_{i j}\right)$. The average link delay solely depends on the traffic. We can categtory the traffic based on their characteristics as a Short Range Dependent (SRD) and Long Range Dependent (LRD). The example of SRD traffics are VoIP traffic, and constant bit rate (CBR) type. When the link traffic shows the behaviour of SRD characteristics, then we can model the link queueing delay as an exponential distribution along with parameter $\mu_{i j}-\lambda_{i j}$. The average network average delay $T_{1}$ can be calculated by using the Little's formula [12]:

$$
T_{1} \cong \frac{1}{\lambda} \sum_{(i, j) \in A}\left[\frac{\lambda_{i j}}{\mu_{i j}-\lambda_{i j}}\right]
$$

On the other hand, the example of LRD traffics include data traffic, and Variable Bit Rate (VBR). The behaviour of link traffic in terms of LRD can be modelled using the fractional Brownian motion ( $\mathrm{fBm}$ ) queueing system. This queueing system has a heavy-tailed Weibull distribution [13]. 


$$
P_{r}\left(Q_{i j}>q\right) \approx \exp \left(-\frac{\left(\mu_{i j}-\lambda_{i j}\right)^{2 H}}{2 \kappa^{2}(H) a \lambda_{i j}} q^{2-2 H}\right)
$$

where $\kappa(H)=H^{H}(1-H)^{1-H}, \quad H \in[0.5,1)$ is the Hurst parameter and $a$ is the index of dispersion. The network average delay $\left(T_{2}\right)$ in case of LRD traffic can be calculated by applying the Little's formula:

$$
T_{2} \cong \tau \cdot \sum_{(i, j) \in A}\left[\frac{\lambda_{i j}}{\left(\mu_{i j}-\lambda_{i j}\right)^{2 H}}\right]^{\frac{1}{2-2 H}}
$$

where $\tau=\Gamma\left(1+\frac{1}{2-2 H}\right)\left[2 \kappa^{2}(H) a\right]^{\frac{1}{2-2 H}}$.

\section{A. QoE Modelling and Measurement}

Quality of Experience (QoE) is subjective by nature, because of its relationship with user's point of view and its own concept of a 'good quality'. The ability to measure QoE would give network operators some sense of the contribution of the network's performance to the overall customer satisfaction, in terms of reliability, availability, scalability, speed, accuracy and efficiency. Many network researchers are now working on this concept, and trying to integrate it in network decisions to ensure a high customer satisfaction with minimum network resources [14]. The Mean Opinion Score (MOS) is an example of a subjective measurement method in which users rate the service quality by giving five different point score from 5 to 1 , where 5 is the best and 1 is the worst quality. MOS is a discrete values, but authors in [15] extend the discrete values and proposed Opinion Score (OS) as a new QoE measurement scale that introduces a new value 0 . According to OS scale, the quality is consider bad when scale is between $\lfloor 0-1]$, poor $\lfloor 1-2]$, fair $\lfloor 2-3]$, good $\lfloor 3-4]$, and excellent $\lfloor 4-5]$. Initially, it is necessary to identify precisely the factors that affect QoE.

The Quality of Service (QoS) mainly influences the UE's QoE, as many QoS parameters directly/indirectly contributed their impact on the end perceived quality of service. The main QoS parameters that affects the multimedia services are bandwidth, jitter, delay and packet loss rate, etc. In case of multimedia video streaming service, the bandwidth can be managed by selecting the suitable video codec, while jitter can be handled by configuring the buffer management of the UE's decoder. However, the delay and packet loss rate are two main QoS parameters that required to be handle in an efficient way in order to improve the user perceived QoE. The delay is an essential parameter that can be caused dropping of video packet. Generally, when a packet is arrived after the end of threshold timer, then a packet does not consider in realtime application (e.g. video streaming), and it is believed to be lost. We shall use the delay and packet loss rate as key QoS parameters in order to compute the user's QoE.

Each link between eNodeB and UE has QoE parameter for each UE, that is computed based on QoS parameters (delay and packet loss rate). We model the each link $(i, j) \in A$ as an arc of graph that represents the UE's QoE based on QoS offered by different eNodeB serving in the region of interest.

The quality of video streaming service, and QoS parameters (delay and packet loss) are correlated via exponential hypothesis. We proposed the QoE model that are based on the exponential hypothesis presented in [16] and [17], to calculate the user's QoE for video service, which is shown by Equation 4

$$
Q \circ E_{(i, j)}=\exp ^{1.576-\left(4.188 \times 10^{-4}\right) \times D-\left(5.766 \times 10^{-2}\right) \times P L R}
$$

where $Q o E_{(i, j)}$ represents the user perception $U E_{i}$ from the $e N o d e B_{j}, D$ represents the delay, and $P L R$ is the packet loss rate on that link. We randomly select the some values of delay and packet loss rate, and evaluate the impact of delay and packet loss rate on user's QoE using proposed method as illustrated in Figure 2.

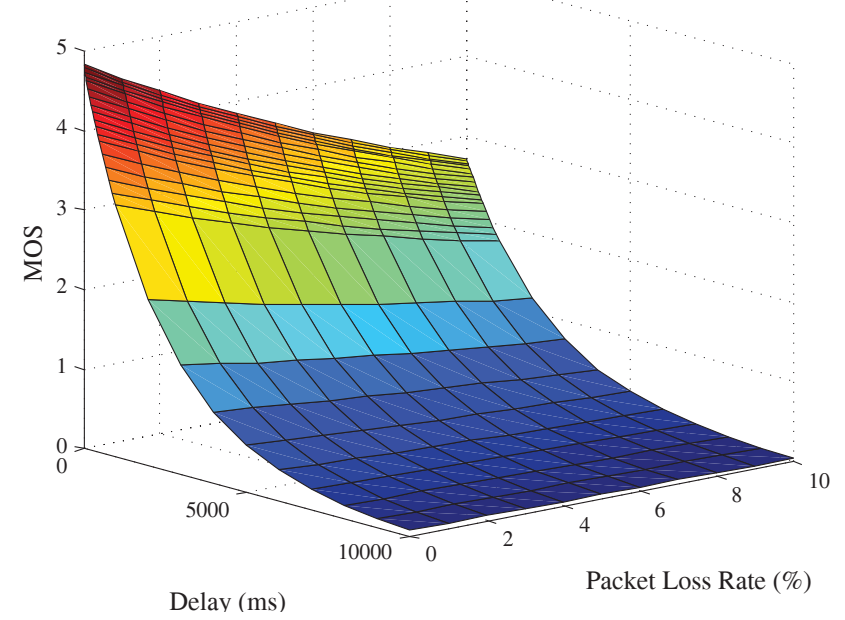

Fig. 2: Impact of Delay and PLR on QoE

\section{OPTIMIZATION MODELS}

\section{A. Network Design}

We consider a network where each arc $(i, j)$ belongs to $A$ has packet sending cost, and each vertex $i$ in $V$ has value represented as $b_{i}$, which depicting the demand or supply of that vertex. The network has set of demands, which is shown by symbol $S$. Each demand $s \in S$ originates from one source node $o_{s} \in V$, it terminates at another node $t_{s} \in V$, and there is an amount of traffic associated with this link, represented as $b_{s}$. We assume that each $\operatorname{arc}(i, j) \in A$ has capacity $\mu(i, j)$, which represents the maximum traffic flow on the arc; however, a lower bound $l(i, j)$ denotes the minimum amount of traffic flow that must flow on the arc. Each node or vertex $i \in V$ has some supply/demand shows as an integer number $b(i)$. In a case, if $b(i)>0$ then node $i$ considers as a supply node; if $b(i)<0$, node is a demand node with a demand of $-b(i)$; and when $b(i)=0$, node will be transshipment node. In our problem, the objective is to minimize the delay of traffic flow that can cause the packet to be lost, and tends to minimize the user's QoE. 
Let's us consider a network, where there are total $N$ links can be established. The problem is formulated as follows:

$$
\min T 1 / T 2
$$

subject to:

$$
\sum_{j:(i, j) \in A} f_{i j}^{s}-\sum_{j:(j, i) \in A} f_{j i}^{s}== \begin{cases}b_{s}, & i=o_{s} \\ -b_{s}, & i=t_{s}, s \in S \\ 0, & \text { otherwise }\end{cases}
$$

$$
\begin{gathered}
l_{i j} \leq f_{i j}^{s} \leq \mu_{i j},(i, j) \in A \\
0 \leq f_{i j}^{s} \leq y_{i j} * b_{s}, \quad s \in S,(i, j) \in A
\end{gathered}
$$

The above constraints can be interpreted as follows:

Constraint 6 can be refereed as mass balance constraints, where link's flow in terms of demand/supply can be represented as $f_{i}^{s}$. The first term in the constraint represents the total outflow of the node (i.e the total flow emitting from the flow), while the second term shows the total inflow of a node (i.e. total flow entering the node). According to mass balance constraint, the supply/demand of the node must equal to the total outflow minus total inflow of that node. When a node is origination of the demand (supply node) then its outflow exceeds its inflow $\left(b_{s}\right)$; if a node is termination of the demand (demand node) then its inflow exceeds its outflow (i.e. $-b_{s}$ ); and if the node is an intermediate (transshipment node) then its outflow is equal to its inflow (i.e. 0).

Constraint 7, refers as flow bound constraints, where flow $f_{i, j}^{s}$ must fulfil the link lower bound and capacity constraints. It bounds the traffic flow within the allowable flows' range. Generally, the lower bounds on arc flows have zero value.

Constraint 8 shows that if there is flow passing on $\operatorname{arc}(i, j)$, then that $\operatorname{arc}(i, j)$ should be selected. The variable $y_{i j}$ represents the link $(i, j)$ is established $(=1)$ or not $(=0)$.

\section{B. $Q o E$}

In cloud computing network, UE's QoE mainly represents the quality providing by the serving server among different cloud servers. The UE's QoE can be calculated based on the QoS parameters (e.g. delay, packet loss rate, etc.) of the traffic flow (e.g. video), that represents the user satisfaction level. We consider a cellular network where the region of interest contains in eNodeBs and serving the available UEs within its coverage area. In future $5 \mathrm{G}$ cellular network, C-RAN will dynamically allocate the radio resource to serve the UE. In this context, UE's satisfaction is very important, because network QoS provided by the server can badly influence the UE's QoE. The user satisfaction will be a key factor in $5 \mathrm{G}$ networks, and giving the power to user for selecting the appropriate server in C-RAN based on user's QoE will successfully achieve this goal.

Let us consider the network coverage has $U E_{i}$ users at location $i$, and it is considered that location must be finite in number. It is necessary for location $i$ that it must has some QoE value in order to assign to eNodeB $j$. Based on current QoS parameters, we consider that QoE is computed before assigning the UE to other eNodeB. The eNodeB $\left(s^{\prime} j\right)$ can serve $u_{j}$ UE. The goal is to assign UE to eNodeB based on the QoE, and minimizes cost associated with the link $(i, j)$. Instead of describe the general model formally, we consider a simple model ingredients based on Figure 3.

Each location $i$ has the node as $d_{i}$ and each cell $j$ represents as node $s_{j}^{\prime}$. The decision variable is the QoE value at location $i$ that is computed from serving QoS parameters from cell $j$, and it is represented by an $\operatorname{arc}(i, j)$ e.g. from node $d_{i}$ to node $s_{j}^{\prime}$. We connect the each $s_{j}^{\prime}$ node to $s_{j}$ node, while flow on the arc must satisfy the lower and upper bounds limit of the $\operatorname{arc}\left(s_{j}^{\prime}, s_{j}\right)$ that is equal to $\left(\lfloor r\rfloor \mu_{j},\lceil r\rceil \mu_{j}\right)$, where $\lfloor r\rfloor$ represents a lower bound and $\lceil r\rceil$ represents the upper bound. Finally, it is necessary to consider the constraint that node $j$ has service capacity $\mu_{j}$.

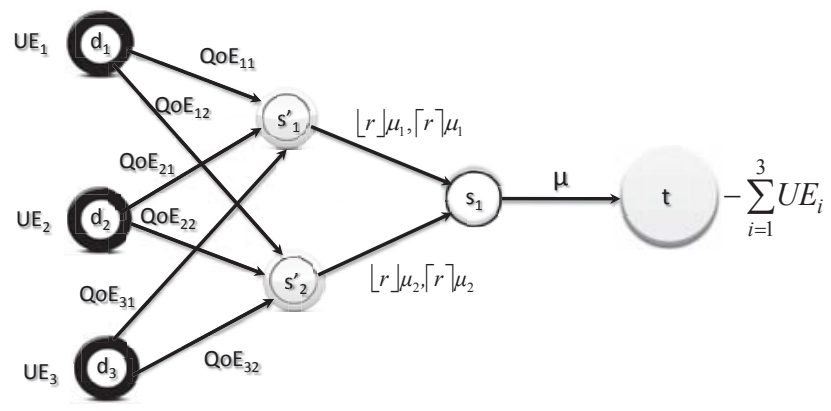

Fig. 3: Network Diagram

\section{RESULTS}

The performance of the proposed QoE method is compared with QoV method [16] to calculate the user's QoE for video streaming along with the described analytical methods for the delay. The network delay is calculated for both SRD (Constant Bit Rate (CBR)) and LRD (Variable Bit Rate (VBR)) traffic models. In case of SRD, we consider the VoIP traffic, while video streaming traffic use for LRD by selecting the $a$ half of link capacity $(a=\mu / 2)$ with $H=0.7$, and $\lambda=693$. The link serving capacity is $\mu=50 \mathrm{Mbps}$ for both the traffic models.

Figure 4, shows the influence of different packet arrival rates on the overall network delay using video streaming traffic. It is observed that when the packet arrival rates increase then delay also increases, but delay value is still very low (less than 1 ms) that does not degrade too much the network performance. One of the key challenges of $5 \mathrm{G}$ network is to keep the network delay less than one millisecond in order to support real time mobile control and vehicle-to-vehicle application and communication. The user's QoE is calculated using the proposed method (see Equation 4) based on these delay values. It is noticed that the overall QoE is always in Excellent scale $(M O S>4)$.

The proposed QoE method is also evaluated by considering the QoS parameters in terms of delay and Packet Loss Rate 


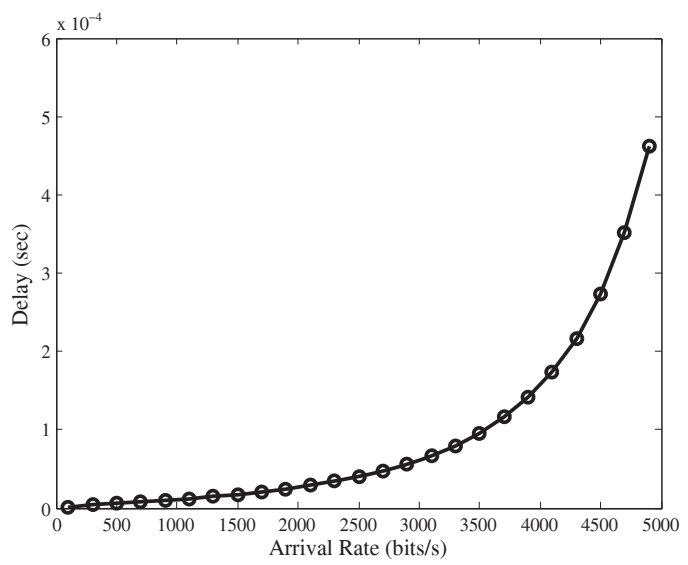

Fig. 4: Video Delay

(PLR). Figure 5 illustrates the impact of delay and PLR on user's QoE using the video streaming traffic. Based on delay values as shown in Figure 4, the user's QoE is always high, and it is approximately $M O S=4.8$ when there is not any PLR. Let's consider that there are some packets are dropped due to limitation of the user device. The possible cases can be buffer (overflow/underflow), insufficient memory, and lack of system resources to process the packets, etc. In these circumstances; the impact of delay and different values of PLR on the user perceived quality experience is measured, and presented in Figure 5. The increases in PLR also decrease the user's QoE, as when $P L R=20 \%$ then user's QoE in terms of value $M O S$ is 1.5 .

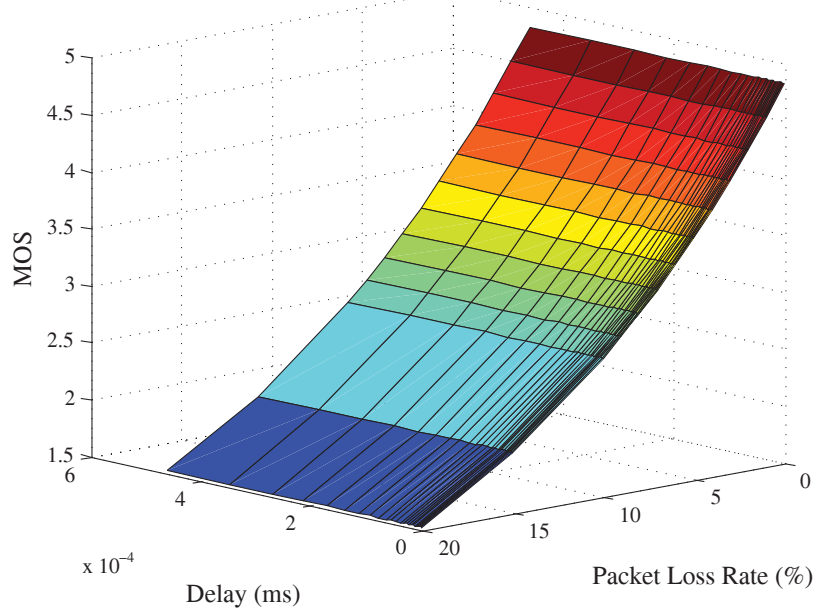

Fig. 5: Proposed QoE Method: Impact of Delay and PLR on QoE

Figure 6, illustrates the impact of delay and packet loss rate on the QoV method using the video streaming traffic. The results clearly shows that QoV method does not properly handle the packet loss rate, because our analytical method reduces the overall network delay as shown in Figure 4. The maximum user's QoE in terms of MOS is 2.8, when the network delay is zero and $P L R$ is also $0 \%$. However, when
$P L R$ increases then it start reducing the user satisfaction, and when $P L R=20 \%$ then user's QoE value is $M O S=1$.

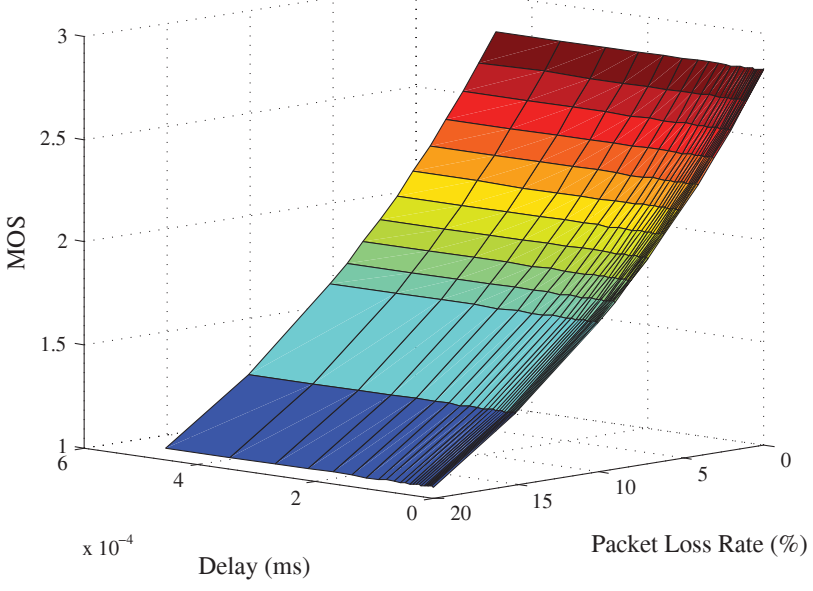

Fig. 6: QoV Method: Impact of Delay and PLR on QoE

Figure 7, depicts an impact of $P L R$ on proposed QoE method and QoV method, when delay is zero. The results clearly shows that proposed QoE method performs better than QoV method. The proposed QoE method has $M O S=4.8$ while QoV has $M O S=2.8$ when $P L R=0 \%$. However, QoV has $M O S=1$ when $P L R=20 \%$, but proposed method has $M O S=1.5$, because high $P L R$ badly affects the user's satisfaction [18].

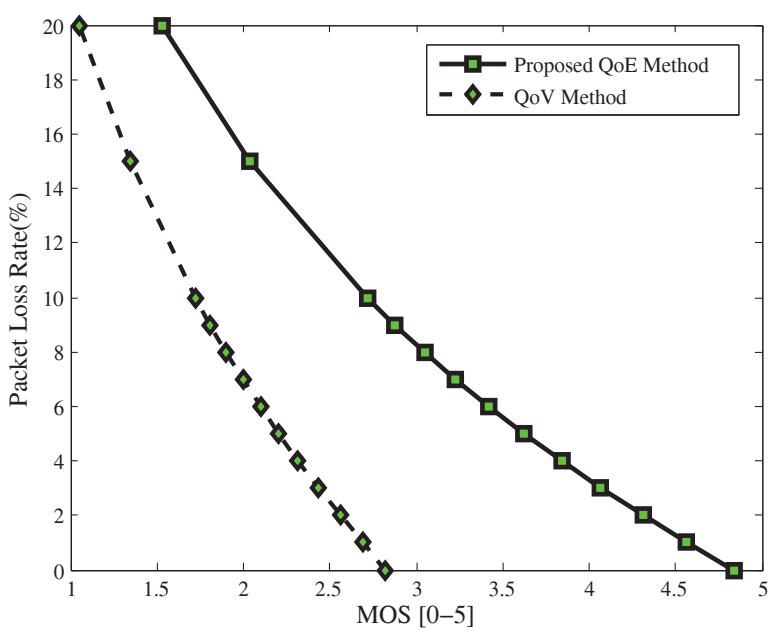

Fig. 7: Proposed QoE method vs QoV Method

Figure 8, shows how the different packet arrival rates affect the overall network delays using the VoIP traffic. The result clearly depicts that increase in packet arrival rate also increases the delay, but still all the delay values are very low that does not significantly decrease the network performance. When the network delay is very low, as in our case, then user's QoE will be at a maximum level for VoIP traffic, because it is a delay sensitive traffic. In LTE-A, the network delay budget for VoIP is 100 ms [19]. Generally; VoIP traffic model generated 
the voice packet after each $20 \mathrm{~ms}$ [20]. According to best of our knowledge, we do not find an appropriate method in the literature to analysis the impact of PLR on user's QoE. The E-model method proposed by ITU-T Rec. G.107 [21] is used to calculate transmission Rating factor $(R-$ factor $)$ for evaluating the voice quality. Based on E-model, the proposed methods in [14], [22], and [23] are not valid for very low delay.

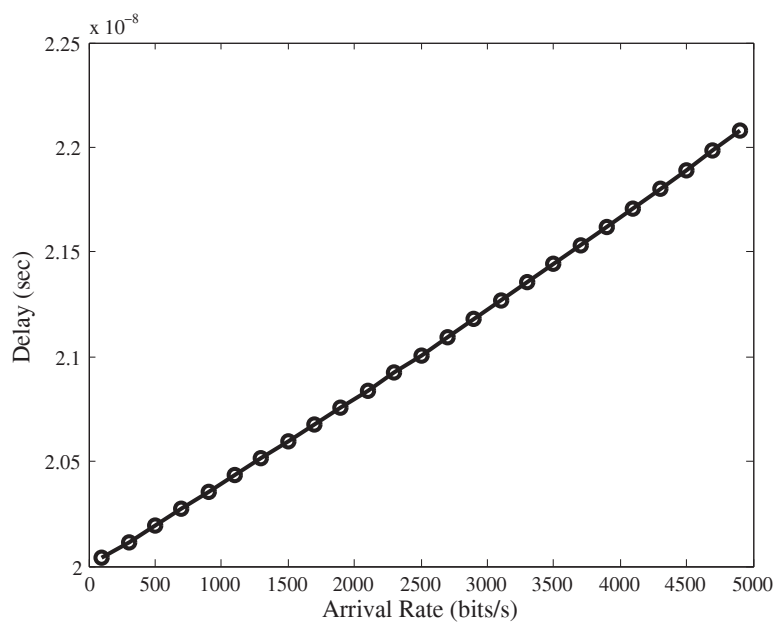

Fig. 8: VoIP Delay

\section{CONCLUSION}

In this paper, we have described a method that minimizes the overall network delay of both multimedia services, the CBR (VoIP) and VBR (video streaming) traffic models. The impact of network delay on user satisfaction is measured by the proposed QoE method. The proposed QoE method calculates the user's QoE for video streaming service, and it is compared with the QoV method. The results clearly show that our analytical method successfully reduces the overall network delay that is less than one millisecond. The low delay significantly improves the user's QoE as it is shown in the results. The performance of proposed QoE method is evaluated by considering the impact of delay and PLR on user perceived quality experience for video streaming service. The proposed QoE method performs better than QoV in terms of delay and PLR.

The VoIP traffic is a delay sensitive service, and our described method effectively reduces the network delay. The proposed methods in the literature are unable to calculate the user's QoE for VoIP because of very low delay. In this context, we can state that user's QoE will be maximum because of very low delay by using our described analytical method. In the future work, we shall propose a QoE measurement method for VoIP in $5 \mathrm{G}$ networks that will also consider energy saving of mobile device.

\section{ACKNOWLEDGMENT}

LISSI Laboratory thanks the CELTIC European Project 'NOTTS' for financial support.

\section{REFERENCES}

[1] Cisco System Inc. Cisco visual networking index: Global mobile data traffic forecast update 2013-2018. 2014.

[2] G. Pallis. Cloud computing: The new frontier of internet computing. Internet Computing, IEEE, 14(5):70-73, Sept 2010.

[3] Yegui Cai, F. Yu, and Shengrong Bu. Cloud computing meets mobile wireless communications in next generation cellular networks. IEEE Network, 28(6):54-59, Nov 2014.

[4] Meng Guo, Mostafa H. Ammar, and Ellen F. Zegura. Selecting among replicated batching video-on-demand servers. In Proceedings of the 12th International Workshop on Network and Operating Systems Support for Digital Audio and Video, NOSSDAV '02, pages 155-163, New York, NY, USA, 2002. ACM

[5] G. Pierre and M. van Steen. Globule: a collaborative content delivery network. IEEE Communications Magazine, 44(8):127-133, Aug 2006.

[6] Xing Zhou, T. Dreibholz, and E.P. Rathgeb. A new server selection strategy for reliable server pooling in widely distributed environments. In IEEE Second International Conference on the Digital Society, pages 171-177, Feb 2008.

[7] Young-Tae Han, Min-Gon Kim, and Hong-Shik Park. A novel server selection method to achieve delay-based fairness in the server palm. IEEE Communications Letters, 13(11):868-870, November 2009.

[8] Hai Anh Tran, S. Hoceini, A. Mellouk, J. Perez, and S. Zeadally. Qoe-based server selection for content distribution networks. IEEE Transactions on Computers, 63(11):2803-2815, Nov 2014.

[9] G. Rubino. Quantifying the Quality of Audio and Video Transmissions over the Internet: the PSQA Approach. 2005.

[10] 5G A Technology Vision. Huawei white paper, access on 18-dec-2014. In Online: (http://www.huawei.com/5gwhitepaper/), 2014.

[11] V. Farkas, B. Heder, and S. Novaczki. A split connection tcp proxy in lte networks. Information and Communication Technologies-Lecture Notes in Computer Science Springer, 7479(10):263-274, 2012.

[12] Leonard Kleinrock. Theory, Volume 1, Queueing Systems. WileyInterscience, 1975.

[13] I. Norros. On the use of fractional brownian motion in the theory of connectionless networks. IEEE Journal on Selected Areas in Communications, 13(6):953-962, Aug 1995.

[14] M.Sajid Mushtaq, Brice Augustin, and Abdelhamid Mellouk. Qoe-based lte downlink scheduler for voip. In IEEE Wireless Communications and Networking Conference (WCNC), pages 2190-2195, April 2014.

[15] M.Fiedler J. Shaikh and D.Collange. Quality of experience from user and network perspectives. Springer Journal Annals of telecommunications -

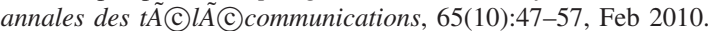

[16] S. Aroussi, T. Bouabana-Tebibel, and A. Mellouk. Empirical qoe/qos correlation model based on multiple parameters for vod flows. In Global Communications Conference (GLOBECOM), 2012 IEEE, pages 19631968, Dec 2012.

[17] M. Fiedler, T. Hossfeld, and P. Tran-Gia. A generic quantitative relationship between quality of experience and quality of service. Network, IEEE, 24(2):36-41, March 2010.

[18] M.S. Mushtaq, B. Augustin, and A. Mellouk. Qoe: User profile analysis for multimedia services. In Communications (ICC), IEEE International Conference on, pages 2289-2294, June 2014.

[19] M.Sajid Mushtaq, Scott Fowler, Abdelhamid Mellouk, and Brice Augustin. Qoe/qos-aware lte downlink scheduler for voip with power saving. International Journal of Network and Computer Applications Elsevier (Accepted), Feb 2014.

[20] 3GPP; LTE Physical Layer Framework for Performance Verification Radio Access Network (RAN). In TSG-RANI no.48, RI-070674, 2007.

[21] International Telecommunication Union (ITU-T); The E model: a computational model for use in transmission planning. In Recommendation G.107, Dec. 2011.

[22] S. Uemura, N. Fukumoto, H. Yamada, and H. Nakamura. QoS/QoE measurement system implemented on cellular phone for NGN. In 5th IEEE Consumer Communications and Networking Conference (CCNC), pages $117-121$, Jan. 2008.

[23] Lijing Ding and R.A. Goubran. Speech quality prediction in voip using the extended e-model. In IEEE Global Telecommunications Conference,(GLOBECOM '03), volume 7, pages 3974-3978 vol.7, 2003. 